\title{
Dynamic Spectrum Management: A Complete Complexity Characterization
}

\author{
Ya-Feng Liu
}

\begin{abstract}
Consider a multi-user multi-carrier communication system where multiple users share multiple discrete subcarriers. To achieve high spectrum efficiency, the users in the system must choose their transmit power dynamically in response to fast channel fluctuations. Assuming perfect channel state information, two formulations for the spectrum management (power control) problem are considered in this paper: the first is to minimize the total transmission power subject to all users' transmission data rate constraints, and the second is to maximize the min-rate utility subject to individual power constraints at each user. It is known in the literature that both formulations of the problem are polynomial time solvable when the number of subcarriers is one and strongly NP-hard when the number of subcarriers are greater than or equal to three. However, the complexity characterization of the problem when the number of subcarriers is two has been missing for a long time. This paper answers this long-standing open question: both formulations of the problem are strongly NP-hard when the number of subcarriers is two.
\end{abstract}

\section{Index Terms}

Complexity theory, multi-carrier communication system, spectrum management, strong NP-hardness.

\section{INTRODUCTION}

In multi-carrier (multi-tone) communication systems, the transmission frequency spectrum is partitioned into a number of orthogonal subcarriers on which parallel data can be simultaneously

Y.-F. Liu is with the State Key Laboratory of Scientific and Engineering Computing, Institute of Computational Mathematics and Scientific/Engineering Computing, Academy of Mathematics and Systems Science, Chinese Academy of Sciences, Beijing, 100190, China (e-mail: yafliu@1sec.cc.ac.cn). This work is partially supported by the National Natural Science Foundation of China under Grants 11671419, 11301516, 11331012, 11631013, and 11571221. 
transmitted without causing interferences with each other. However, different users interfere with each other on the same subcarrier. The common examples of multi-carrier systems include wireless orthogonal frequency division multiplex (OFDM) systems (such as the 802.11) and wireline discrete multi-tone (DMT) systems (such as the digital subscriber line (DSL) system). In both of OFDM and DMT systems, a pair of discrete Fourier transform (DFT) and inverse discrete Fourier transform (IDFT) is used to effectively decompose the frequency-selective wideband channel into a group of non-selective narrowband subcarriers, which makes them robust against large delay spreads by preserving orthogonality in the frequency domain [1], [2].

Spectrum management, also called spectrum balancing or power control, is a central issue in the design of interference-limited multi-user multi-carrier communication systems. This is because in such systems the achievable data rate of each user depends not only on its own power allocation but also on the power allocation of all other users. The spectrum management problem in multi-user multi-carrier communication systems is often formulated as an optimization problem such as the system utility maximization problem subject to power budget constraints or the total power minimization problem subject to Quality-of-Service (QoS) constraints.

The spectrum management problem in the interference-limited multi-user multi-carrier communication system has been extensively studied; see [3]-[22] and references therein. The authors of [3] showed that the problem (under various optimization models) is (strongly) NP-hard when the number of subcarriers is greater than or equal to three. They also identified several subclasses of the problem which are polynomial time solvable when the number of subcarriers is one, such as the min-rate utility maximization problem and the total power minimization problem. However, the complexity characterization of the problem for the case where the number of subcariers is two was missed for a long time in the literature. This might be due to the following two reasons.

- The standard way to prove an optimization problem is NP-hard is to establish a polynomial time reduction from a known NP-complete problem to its corresponding feasibility problem or decision problem [23]-[26]. Since there are a large number of NP-complete problems involving "three", one has a lot of choices to pick an NP-complete problem involving "three" (as did in [3]), establish a polynomial time reduction from it to the spectrum management problem with three subcarriers, and show that the problem is NP-hard. In contrast, there are few NP-complete problems involving "two" and this makes it hard to show the NPhardness of the spectrum management problem when the number of subcarriers is two. 
In fact, there is usually a complexity gap between problems involving "two" and "three", i.e., 3-SATISFIABILITY, 3-DIMENSIONAL MATCHING, and 3-COLORABILITY problems are all NP-complete but 2-SATISFIABILITY, 2-DIMENSIONAL MATCHING, and 2-COLORABILITY problems are all polynomial time solvable.

- Exploring the complexity of an optimization problem typically involves switching back and forth between trying to develop a polynomial time algorithm for the problem and trying to prove it NP-hard, until one of them succeeds. Since many problems involving "two" are polynomial time solvable (as mentioned above), this might give a wrong sense that the spectrum management problem is polynomial time solvable when the number of subcarriers is two, which makes it hard to characterize the complexity of the problem.

The complexity results in [3] suggest that there is no polynomial time algorithms which can solve the general spectrum management problem to global optimality (unless $\mathrm{P}=\mathrm{NP}$ ), and determining an approximately optimal or locally optimal spectrum management strategy in polynomial time is more realistic in practice (especially when a very fast responsiveness is required [15]). Therefore, various (heuristic) algorithms [3]-[21], including iterative water-filling algorithms, dual decomposition algorithms, and successive convex/concave approximation algorithms, have been proposed for solving the problem.

In this paper, we focus on the characterization of the computational complexity status of the spectrum management problem for the multi-user multi-carrier communication system. In particular, we consider two formulations of the problem. The first one is the problem of minimizing the total transmission power subject to all users' QoS constraints. The second one is the problem of maximizing the minimum rate among all users while respecting the total transmission power constraint of each user. The main contribution of this paper is to answer a long-standing open question: both aforementioned formulations of the spectrum management problem are strongly NP-hard when the number of subcarriers is two. The developed techniques in this paper can be extended to show the (strong) NP-hardness of other related optimization problems involving "two" arising from signal processing and wireless communications.

\section{PRoblem Formulation}

Consider a multi-user multi-carrier communication system, where there are $K$ users (transmitterreceiver pairs) sharing $N$ discrete subcarriers. Denote the set of users and the set of subcarriers 
by $\mathcal{K}=\{1,2, \ldots, K\}$ and $\mathcal{N}=\{1,2, \ldots, N\}$, respectively. For any $k \in \mathcal{K}$ and $n \in \mathcal{N}$, suppose $s_{k}^{n} \in \mathbb{C}$ to be the symbol that transmitter $k$ wishes to send to receiver $k$ on subcarrier $n$, then the received signal $\hat{s}_{k}^{n}$ at receiver $k$ on subcarrier $n$ can be expressed by

$$
\hat{s}_{k}^{n}=\sum_{j \in \mathcal{K}} h_{k, j}^{n} s_{j}^{n}+z_{k}^{n},
$$

where $h_{k, j}^{n} \in \mathbb{C}$ is the channel coefficient between the $j$-th transmitter and the $k$-th receiver on subcarrier $n$ and $z_{k}^{n} \in \mathbb{C}$ is the additive white Gaussian noise (AWGN) with distribution $\mathcal{C N}\left(0, \eta_{k}^{n}\right)$. Denoting the power of $s_{k}^{n}$ by $p_{k}^{n}$; i.e., $p_{k}^{n}:=\left|s_{k}^{n}\right|^{2}$, the received power at receiver $k$ on subcarrier $n$ is given by

$$
\sum_{j \in \mathcal{K}} g_{k, j}^{n} p_{j}^{n}+\eta_{k}^{n}, k \in \mathcal{K}, n \in \mathcal{N},
$$

where $g_{k, j}^{n}:=\left|h_{k, j}^{n}\right|^{2}$ stands for the channel gain between the $j$-th transmitter and the $k$-th receiver on subcarrier $n$. Treating interference as noise, we can write the SINR of receiver $k$ on subcarrier $n$ as

$$
\operatorname{SINR}_{k}^{n}=\frac{g_{k, k}^{n} p_{k}^{n}}{\sum_{j \neq k} g_{k, j}^{n} p_{j}^{n}+\eta_{k}^{n}}, k \in \mathcal{K}, n \in \mathcal{N},
$$

and transmitter $k$ 's achievable data rate $R_{k}$ (nats/sec) as

$$
R_{k}=\sum_{n \in \mathcal{N}} \ln \left(1+\frac{g_{k, k}^{n} p_{k}^{n}}{\sum_{j \neq k} g_{k, j}^{n} p_{j}^{n}+\eta_{k}^{n}}\right), k \in \mathcal{K} .
$$

In this paper, we consider the following two formulations of the spectrum management problem:

$$
\begin{array}{ll}
\min _{\left\{p_{k}^{n}\right\}} & \sum_{k \in \mathcal{K}} \sum_{n \in \mathcal{N}} p_{k}^{n} \\
\text { s.t. } & R_{k} \geq \gamma_{k}, k \in \mathcal{K}, \\
& \sum_{n \in \mathcal{N}} p_{k}^{n} \leq \bar{p}_{k}, k \in \mathcal{K}, \\
& p_{k}^{n} \geq 0, k \in \mathcal{K}, \quad n \in \mathcal{N},
\end{array}
$$

and

$$
\begin{array}{ll}
\max _{\left\{p_{k}^{n}\right\}} & \min _{k \in \mathcal{K}}\left\{R_{k}\right\} \\
\text { s.t. } & \sum_{n \in \mathcal{N}} p_{k}^{n} \leq \bar{p}_{k}, k \in \mathcal{K}, \\
& p_{k}^{n} \geq 0, k \in \mathcal{K}, n \in \mathcal{N},
\end{array}
$$


where $\gamma_{k}$ is the desired transmission rate target of user $k$ and $\bar{p}_{k}$ is the power budget of transmitter $k$. Formulation (2) minimizes the total transmission power of all users on all subcarriers and formulation (3) maximizes the minimum transmission rate among all users.

\section{COMPLEXITy ANALYSIS}

In this section, we first briefly introduce complexity theory in Section $\amalg-A$ Then, we review existing complexity results of problems (2) and (3) and show that both problems are strongly NP-hard when the number of subcarriers is two in Section III-B. Finally, we extend the developed techniques to show the (strong) NP-hardness of other related optimization problems involving "two" arising from signal processing and wireless communications in Section $\amalg-C$.

\section{A. A Brief Introduction to Complexity Theory}

In computational complexity theory [23]-[26], a problem is said to be NP-hard if it is at least as hard as any problem in the class NP (problems that are solvable in Nondeterministic Polynomial time). NP-complete problems are the hardest problems in NP in the sense that if any NP-complete problem is solvable in polynomial time, then each problem in NP is solvable in polynomial time. A problem is strongly NP-hard (strongly NP-complete) if it is NP-hard (NPcomplete) and it cannot be solved by a pseudo-polynomial time algorithm. An algorithm that solves a problem is called a pseudo-polynomial time algorithm if its time complexity function is bounded above by a polynomial function related to both of the length and the numerical values of the given data of the problem. This is in contrast to the polynomial time algorithm whose time complexity function depends only on the length of the given data of the problem. It is widely believed that there can not exist a polynomial time algorithm to solve any NP-complete, NP-hard, or strongly NP-hard problem (unless $\mathrm{P}=\mathrm{NP}$ ).

The standard way to prove an optimization problem is NP-hard is to establish the NP-hardness of its corresponding feasibility problem or decision problem. The latter is the problem to decide if the global minimum (maximum) of the optimization problem is below (above) a given threshold or not. To show a decision problem $\mathcal{P}_{2}$ is NP-hard, we usually follow three steps: 1) choose a suitable NP-complete decision problem $\mathcal{P}_{1} ; 2$ ) construct a polynomial time transformation from any instance of $\mathcal{P}_{1}$ to an instance of $\mathcal{P}_{2} ; 3$ ) prove under this transformation that any instance of 
problem $\mathcal{P}_{1}$ is true if and only if the constructed instance of problem $\mathcal{P}_{2}$ is true. See [23]-[26] for more on complexity theory.

\section{B. Strong NP-Hardness of Problems (2) and (3) when $N=2$}

Both problems (2) and (3) are polynomial time solvable when $N=1$. More specifically, when $N=1$, problem (2) is equivalent to

$$
\begin{array}{ll}
\min _{\left\{p_{k}\right\}} & \sum_{k \in \mathcal{K}} p_{k} \\
\text { s.t. } & g_{k, k} p_{k} \geq\left(\exp \left(\gamma_{k}\right)-1\right)\left(\sum_{j \neq k} g_{k, j} p_{j}+\eta_{k}\right), k \in \mathcal{K}, \\
& \bar{p}_{k} \geq p_{k} \geq 0, k \in \mathcal{K},
\end{array}
$$

which is a linear program (solvable in polynomial time). When $N=1$, problem (3) reduces to

$$
\begin{array}{ll}
\max _{\tau,\left\{p_{k}\right\}} & \tau \\
\text { s.t. } & g_{k, k} p_{k} \geq \tau\left(\sum_{j \neq k} g_{k, j} p_{j}+\eta_{k}\right), k \in \mathcal{K}, \\
& \bar{p}_{k} \geq p_{k} \geq 0, k \in \mathcal{K},
\end{array}
$$

which is polynomial time solvable by using a binary search on $\tau$; see [3, Theorem 2]. In fact, both problems (2) and (3) are also polynomial time solvable (by the water-filling algorithm) when $K=1$ (i.e., there is only a single user in the system) [5, Theorem 4.1].

Problems (2) and (3) become computationally intractable when the number of subcarriers is greater than or equal to three. In particular, it is shown in [3, Theorem 2] that problem (2) is strongly NP-hard when $N \geq 3$. By using the same argument as in the proof of [3, Theorem 2], one can also show the strong NP-hardness of problem (3) with $N \geq 3$. However, the complexity characterization of problems (2) and (3) with $N=2$ has been missing for a long time in the literature. In this subsection, we answer this open question and show that both of problems (2) and (3) remain strongly NP-hard when $N=2$.

The NP-hardness proof of problems (2) and (3) for the case $N=2$ is based on a polynomial time reduction from the MAX-2UNANIMITY problem, which was first introduced in [27]. To describe the problem, we first define the UNANIMITY property of a disjunctive clause. Recall that for a given set of Boolean variables, a literal is defined as either a Boolean variable or its 
negation, while a disjunctive clause refers to a logical expression consisting of the logical "OR" of literals.

Definition 1 (UNANIMOUS): For a given truth assignment to a set of Boolean variables, a disjunctive clause is said to be unanimous if all literals in the clause have the same value (whether it is the True or the False value).

Definition 2 (MAX-2UNANIMITY): Given a positive integer $M$ and $m$ disjunctive clauses defined over $n$ Boolean variables, where the number of literals in each clause is 2 , the MAX2UNANIMITY problem is to check whether there exists a truth assignment such that the number of unanimous disjunctive clauses is at least $M$.

Example 1: Given Boolean variables $x_{1}, x_{2}, x_{3}, x_{4}$, define $c_{1}=x_{1} \vee \bar{x}_{2}, c_{2}=x_{1} \vee x_{3}$, $c_{3}=\bar{x}_{2} \vee \bar{x}_{4}, c_{4}=\bar{x}_{3} \vee x_{4}$. All of $x_{1}, x_{2}, x_{3}, x_{4}$ and their negations $\bar{x}_{1}, \bar{x}_{2}, \bar{x}_{3}, \bar{x}_{4}$ are literals; all of $c_{1}, c_{2}, c_{3}, c_{4}$ are disjunctive clauses; if we set $x_{1}=1, x_{2}=0, x_{3}=1, x_{4}=0$, then all of $c_{1}, c_{2}, c_{3}, c_{4}$ are unanimous (or satisfied unanimously); the clauses $c_{1}, c_{2}, c_{3}, c_{4}$ defined on $x_{1}, x_{2}, x_{3}, x_{4}$ along with some given positive $M$ is an instance of the MAX-2UNANIMITY problem.

Lemma 1 ([27]): MAX-2UNANIMITY problem is NP-complete.

We are now ready to prove our main results.

Theorem 1: Problem (2) is strongly NP-hard when $N=2$.

Proof: Given any instance of the MAX-2UNANIMITY problem with clauses $c_{1}, c_{2}, \ldots, c_{m}$ defined over Boolean variables $x_{1}, x_{2}, \ldots, x_{n}$ and an integer $M$, we construct below a multi-user multi-carrier interference channel with $2 n+m$ users and 2 subcarriers, where the Boolean variable $x_{i}(i=1,2, \ldots, n)$ corresponds to a pair of users, including user $i$ (called "variable user") and user $n+i$ (called "auxiliary variable user"); each clause $c_{j}(j=1,2, \ldots, m)$ corresponds to user $2 n+j$ (called "clause user"). Hence, $\mathcal{K}=\{1,2, \ldots, 2 n+m\}$ and $\mathcal{N}=\{1,2\}$.

Next, we construct channel parameters for all $2 n+m$ users on 2 subcarriers. Before going into very details, let us first give a high level preview of the construction. More specifically, we first construct channel parameters of all users associated with Boolean variables (i.e., "variable users" and "auxiliary variable users") such that the only ways for each pair of the users to satisfy their transmission rate requirements are that one user transmits full power on one subcarrier and the other user transmits full power on the other subcarrier. Then, we construct channel parameters of all users associated with clauses (i.e., "clause users") such that each "clause user" suffers 
interferences from only two "variable users" and/or "auxiliary variable users", whose types (i.e., "variable user" or "auxiliary variable user") and indices are determined by the two literals appearing in the corresponding clause. In addition, we construct channel parameters of "clause users" such that the required total transmission power for the user to satisfy its transmission rate constraint when the corresponding clause is unanimous is strictly less than the one when the corresponding clause is not. In this way, the total transmission power for all users to satisfy their transmission rate constraints depends on the number of unanimous clauses and less total transmission power is needed if and only if more clauses are satisfied unanimously.

We now construct the direct-link and crosstalk channel gains among these $2 n+m$ users on 2 subcarriers. The direct-link channel gains of all users on two subcarriers are set to be

$$
g_{k, k}^{1}=g_{k, k}^{2}=1, k \in \mathcal{K}
$$

The corresponding crosstalk channel gains on 2 subcarriers are: for user $k=1,2, \ldots, n$, set

$$
g_{k, n+k}^{1}=g_{k, n+k}^{2}=1 \text { and } g_{k, \ell}^{1}=g_{k, \ell}^{2}=0, \forall \ell \in \mathcal{K} \backslash\{k, n+k\}
$$

for user $k=n+1, n+2, \ldots, 2 n$, set

$$
g_{k, k-n}^{1}=g_{k, k-n}^{2}=1 \text { and } g_{k, \ell}^{1}=g_{k, \ell}^{2}=0, \forall \ell \in \mathcal{K} \backslash\{k-n, k\}
$$

for user $k=2 n+1,2 n+2, \ldots, 2 n+m$, set $g_{k, \ell}^{1}=g_{k, \ell}^{2}=0$ for all $\ell \in \mathcal{K}$ except

$$
\begin{cases}g_{k, \ell}^{1}=g_{k, \ell}^{2}=1, & \text { if } x_{\ell} \text { appears in } c_{k-2 n} ; \\ g_{k, n+\ell}^{1}=g_{k, n+\ell}^{2}=1, & \text { if } \bar{x}_{\ell} \text { appears in } c_{k-2 n} .\end{cases}
$$

Set $\eta_{k}^{n}=1$ for all $k \in \mathcal{K}$ and $n \in \mathcal{N}$. Then, the transmission rate expressions of all users are: for $i=1,2, \ldots, n$,

$$
R_{i}=\ln \left(1+\frac{p_{i}^{1}}{1+p_{n+i}^{1}}\right)+\ln \left(1+\frac{p_{i}^{2}}{1+p_{n+i}^{2}}\right)
$$

and

$$
R_{n+i}=\ln \left(1+\frac{p_{n+i}^{1}}{1+p_{i}^{1}}\right)+\ln \left(1+\frac{p_{n+i}^{2}}{1+p_{i}^{2}}\right)
$$




$$
\begin{aligned}
& \text { for } j=1,2, \ldots, m, \\
& R_{2 n+j}=\left\{\begin{array}{l}
\ln \left(1+\frac{p_{2 n+j}^{1}}{1+p_{i_{1}}^{1}+p_{i_{2}}^{1}}\right)+\ln \left(1+\frac{p_{2 n+j}^{2}}{1+p_{i_{1}}^{2}+p_{i_{2}}^{2}}\right), \text { if } c_{j}=x_{i_{1}} \vee x_{i_{2}} ; \\
\ln \left(1+\frac{p_{2 n+j}^{1}}{1+p_{i_{1}}^{1}+p_{n+i_{2}}^{1}}\right)+\ln \left(1+\frac{p_{2 n+j}^{2}}{1+p_{i_{1}}^{2}+p_{n+i_{2}}^{2}}\right), \text { if } c_{j}=x_{i_{1}} \vee \bar{x}_{i_{2}} ; \\
\ln \left(1+\frac{p_{2 n+j}^{1}}{1+p_{n+i_{1}}^{1}+p_{i_{2}}^{1}}\right)+\ln \left(1+\frac{p_{2 n+j}^{2}}{1+p_{n+i_{1}}^{2}+p_{i_{2}}^{2}}\right), \text { if } c_{j}=\bar{x}_{i_{1}} \vee x_{i_{2}} ; \\
\ln \left(1+\frac{p_{2 n+j}^{1}}{1+p_{n+i_{1}}^{1}+p_{n+i_{2}}^{1}}\right)+\ln \left(1+\frac{p_{2 n+j}^{2}}{1+p_{n+i_{1}}^{2}+p_{n+i_{2}}^{2}}\right), \text { if } c_{j}=\bar{x}_{i_{1}} \vee \bar{x}_{i_{2}} .
\end{array}\right.
\end{aligned}
$$

In the above, each user $k$ is associated with two variables $p_{k}^{1}$ and $p_{k}^{2}$ for $k \in \mathcal{K}$; each "variable user" $i$ suffers interference from "auxiliary variable user" $n+i$ on both subcarriers 1 and 2 ; each "auxiliary variable user" $n+i$ suffers interference from "variable user" $i$ on both subcarriers 1 and 2 ; each "clause user" $2 n+j$ suffers interference from "variable user" $i_{1}$ and $i_{2}$ and/or "auxiliary variable user" $n+i_{1}$ and $n+i_{2}$, where $c_{j}$ contains literals of $x_{i_{1}}$ and $x_{i_{2}}$. To make the construction of transmission rate expressions clear, an illustrative example is given in Appendix B.

Moreover, let $\gamma_{k}=\ln 2$ and $\bar{p}_{k}=1$ for all $k \in \mathcal{K}$. Then, the constructed instance of problem (2) is

$$
\begin{array}{ll}
\min _{\left\{p_{k}^{n}\right\}} & \sum_{k \in \mathcal{K}} \sum_{n \in \mathcal{N}} p_{k}^{n} \\
\text { s.t. } & R_{k} \geq \ln 2, k \in \mathcal{K}, \\
& p_{k}^{1}+p_{k}^{2} \leq 1, k \in \mathcal{K}, \\
& p_{k}^{n} \geq 0, k \in \mathcal{K}, n \in \mathcal{N},
\end{array}
$$

where $R_{k}$ are given in (6), (7), and (8). The variable correspondence between the MAX2UNANIMITY problem and problem (9) is listed as Table I.

We claim that the transformation from the MAX-2UNANIMITY problem to problem (9) can be performed in polynomial time. The number of users and the number of subcarriers in problem (9) are $2 n+m$ and 2, respectively. Hence, the size of problem (9) is bounded above by a polynomial (linear) function of the size of the MAX-2UNANIMITY instance. Moreover, the construction of channel parameters/transmission rate expressions for all $2 n+m$ users is straightforward, i.e., for $i=1,2, \ldots, n$, transmission rate expressions of users $i$ and $n+i$ (associated with variable i) are explicitly given in (6) and (7); and for $j=1,2, \ldots, m$, transmission rate expression of 
TABLE I

VAriable Correspondence between MAX-2UNANimity Problem And Problem (9)

\begin{tabular}{ll}
\hline MAX-2UNANIMITY problem & Problem 9 \\
\hline$m$ clauses defined over $n$ variables & $2 n+m$ users communicate over 2 subcarriers \\
Boolean variable $x_{i}$ & power allocation variables $p_{i}^{1}, p_{i}^{2} p_{n+i}^{1}, p_{n+i}^{2}$ of users $i$ and $n+i$ \\
clause $c_{j}$ & transmission rate $R_{2 n+j}$ in 8 of user $2 n+j$ \\
literal $x_{i}$ appears in clause $c_{j}$ & user $i$ causes interference $p_{i}^{1}$ and $p_{i}^{2}$ to user $2 n+j$ on 2 subcarriers \\
literal $\bar{x}_{i}$ appears in clause $c_{j}$ & user $n+i$ causes interference $p_{n+i}^{1}$ and $p_{n+i}^{2}$ to user $2 n+j$ on 2 subcarriers \\
\hline
\end{tabular}

user $2 n+j$ (associated with clause $j$ ) is explicitly given in (8). Therefore, the transformation from the MAX-2UNANIMITY problem to problem (9) can be performed in polynomial time.

Next, we show that there exists a truth assignment such that at least $M$ clauses are satisfied unanimously for the given MAX-2UNANIMITY instance if and only if the optimal value of problem (9) is less than or equal to $2 n+M+4(\sqrt{2}-1)(m-M)$.

If there exists a truth assignment such that $M$ clauses in the MAX-2UNANIMITY problem are unanimous, we claim that the optimal value of problem (9) is less than or equal to $2 n+$ $M+4(\sqrt{2}-1)(m-M)$. Let $\left\{x_{i}\right\}$ be the truth assignment such that $M$ clauses are unanimous in the MAX-2UNANIMITY problem. We set

$$
p_{i}^{1}=p_{n+i}^{2}=1-x_{i}, p_{i}^{2}=p_{n+i}^{1}=x_{i}, i=1,2, \ldots, n .
$$

With this, we can simply check that $R_{k} \geq \ln 2$ for all $k=1,2, \ldots, 2 n$. Furthermore, we consider transmission rate requirements of the "clause variable" $2 n+j$ with $j=1,2, \ldots, m$.

- If the clause $c_{j}$ is unanimous, then we have either

$$
R_{2 n+j}=\ln \left(1+p_{2 n+j}^{1}\right)+\ln \left(1+\frac{p_{2 n+j}^{2}}{3}\right)
$$

or

$$
R_{2 n+j}=\ln \left(1+\frac{p_{2 n+j}^{1}}{3}\right)+\ln \left(1+p_{2 n+j}^{2}\right) .
$$

In either cases, we can use a total transmission power of 1 to make $R_{2 n+j} \geq \ln 2$ satisfied (by setting $\left(p_{2 n+j}^{1}, p_{2 n+j}^{2}\right)^{T}=(1,0)^{T}$ in the former case and $\left(p_{2 n+j}^{1}, p_{2 n+j}^{2}\right)^{T}=(0,1)^{T}$ in the latter case). 
- If the clause $c_{j}$ is not unanimous, then we must have

$$
R_{2 n+j}=\ln \left(1+\frac{p_{2 n+j}^{1}}{2}\right)+\ln \left(1+\frac{p_{2 n+j}^{2}}{2}\right) .
$$

In this case, we can use a total transmission power of $4(\sqrt{2}-1)$ to make $R_{2 n+j} \geq \ln 2$ satisfied (by setting $p_{2 n+j}^{1}=p_{2 n+j}^{2}=2(\sqrt{2}-1)$ ).

As a result, if there exists a truth assignment such that at least $M$ clauses are satisfied unanimously, then the optimal value of problem (96) is less than or equal to $2 n+M+4(\sqrt{2}-1)(m-M)$.

For the converse part, assuming that the optimal value of problem (9) is less than or equal to $2 n+M+4(\sqrt{2}-1)(m-M)$, we claim that at least $M$ clauses can be made unanimous. It follows from Lemma 2 in Appendix $₫$ that, for $i=1,2, \ldots, n$, the optimal solution of problem (9) must be

$$
\left(p_{i}^{1}, p_{i}^{2}, p_{n+i}^{1}, p_{n+i}^{2}\right)^{T}=(1,0,0,1)
$$

or

$$
\left(p_{i}^{1}, p_{i}^{2}, p_{n+i}^{1}, p_{n+i}^{2}\right)^{T}=(0,1,1,0) .
$$

This, together with (8), implies that the received total interferences at user $2 n+j$ must be exactly 2 for all $j=1,2, \ldots, m$. More specifically, there might be two cases:

- Case 1: the received interference at user $2 n+j$ is equal 2 on one subcarrier and is equal to 0 on the other one;

- Case 2: the received interference at user $2 n+j$ is equal to 1 on both subcarriers.

If Case 1 happens for user $2 n+j$, then the required total transmission power satisfying $R_{2 n+j} \geq$ $\ln 2$ is at least 1 ; while if Case 2 happens for user $2 n+j$, then the required total transmission power satisfying $R_{2 n+j} \geq \ln 2$ is at least $4(\sqrt{2}-1)$. By the assumption that the optimal value of problem (9) is less than or equal to $2 n+M+4(\sqrt{2}-1)(m-M)$, we know that Case 1 must happen at least $M$ times (Case 2 cannot happen more than $m-M$ times). Moreover, it can be checked that

$$
x_{i}=1-p_{i}^{1}, i=1,2, \ldots, n
$$

is a truth assignment which makes at least $M$ clauses in the MAX-2UNANIMITY problem satisfied unanimuously. 
Since the MAX-2UNANIMITY problem is NP-complete (cf. Lemma 1), we conclude that the problem of checking the optimal value of problem (9) is less than or equal to $2 n+M+4(\sqrt{2}-$ 1) $(m-M)$ is strongly NP-hard. Hence, problem (2) is strongly NP-hard.

Theorem 2: Problem (3) is strongly NP-hard when $N=2$.

Proof: The basic idea of proving the strong NP-hardness of problem (3) is to establish a polynomial time reduction from the MAX-2UNANIMITY problem to it. Since this proof is similar to the one of Theorem 1 we just give the proof outline. Given any instance of the MAX2UNANIMITY problem with clauses $c_{1}, c_{2}, \ldots, c_{m}$ defined over Boolean variables $x_{1}, x_{2}, \ldots, x_{n}$ and an integer $M$, we construct below a multi-user multi-carrier interference channel with $2 n+$ $2 m+1$ users and 2 subcarriers. In addition to "variable user" $i$ and "auxiliary variable user" $n+i$ for $i=1,2, \ldots, n$ and "clause user" $2 n+j$ for $j=1,2, \ldots, m$, we also construct "auxiliary clause user" $2 n+m+j$ for $j=1,2, \ldots, m$ and "super user" $2 n+2 m+1$. Hence, $\mathcal{K}=\{1,2, \ldots, 2 n+2 m+1\}$ and $\mathcal{N}=\{1,2\}$.

The difference between the above constructed setup and the one constructed in Theorem 1 is $m$ "auxiliary clause users" and 1 "super user". The purpose of constructing these $m+1$ users is to establish the reduction from the MAX-2UNANIMITY problem to problem (3). More specifically, we construct channel parameters of all "auxiliary clause users" such that they and those of "clause users" are symmetric, i.e., if "clause user" $2 n+j$ suffers interferences from "variable user" $i_{1}$ ("auxiliary variable user" $n+i_{1}$ ) on subcarrier 1 and "auxiliary variable user" $n+i_{2}$ ("variable user" $i_{2}$ ) on subcarrier 2 , then "auxiliary clause user" $2 n+m+j$ suffers interferences from "auxiliary variable user" $n+i_{1}$ ("variable user" $i_{1}$ ) on subcarrier 1 and "variable user" $i_{2}$ ("auxiliary variable user" $n+i_{2}$ ) on subcarrier 2 . Then, we construct channel parameters of the "super user" such that all "clause users" and all "auxiliary clause users" cause equal interferences to the "super user" on two subcarriers. In this way, since channel parameters of all "clause users" and "auxiliary clause users" are symmetric, the "super user" can achieve a higher transmission rate if and only if "clause users" transmit less total power and cause less total interferences to it. Furthermore, it follows from the proof of Theorem 1 that "clause users" transmit less total power if and only if more clauses are satisfied unanimously in the given MAX-2UNANIMITY instance. This finishes the desirable reduction.

We construct a special interference channel with $2 n+m$ users and 2 subcarriers such that transmission rate expressions of all users take the following forms: for $i=1,2, \ldots, n, R_{i}$ and 
$R_{n+i}$ are the same as the ones in (6) and (7), respectively; for $j=1,2, \ldots, m, R_{2 n+j}$ is the same as the one in (8) and

$$
R_{2 n+m+j}=\left\{\begin{array}{l}
\ln \left(1+\frac{p_{2 n+m+j}^{1}}{1+p_{n+i_{1}}^{1}+p_{n+i_{2}}^{1}}\right)+\ln \left(1+\frac{p_{2 n+m+j}^{2}}{1+p_{n+i_{1}}^{2}+p_{n+i_{2}}^{2}}\right), \text { if } c_{j}=x_{i_{1}} \vee x_{i_{2}} ; \\
\ln \left(1+\frac{p_{2 n+m+j}^{1}}{1+p_{n+i_{1}}^{1}+p_{i_{2}}^{1}}\right)+\ln \left(1+\frac{p_{2 n+m+j}^{2}}{1+p_{n+i_{1}}^{2}+p_{i_{2}}^{2}}\right), \text { if } c_{j}=x_{i_{1}} \vee \bar{x}_{i_{2}} ; \\
\ln \left(1+\frac{p_{2 n+m+j}^{1}}{1+p_{i_{1}}^{1}+p_{n+i_{2}}^{1}}\right)+\ln \left(1+\frac{p_{2 n+m+j}^{2}}{1+p_{i_{1}}^{2}+p_{n+i_{2}}^{2}}\right), \text { if } c_{j}=\bar{x}_{i_{1}} \vee x_{i_{2}} ; \\
\ln \left(1+\frac{p_{2 n+m+j}^{1}}{1+p_{i_{1}}^{1}+p_{i_{2}}^{1}}\right)+\ln \left(1+\frac{p_{2 n+m+j}^{2}}{1+p_{i_{1}}^{2}+p_{i_{2}}^{2}}\right), \text { if } c_{j}=\bar{x}_{i_{1}} \vee \bar{x}_{i_{2}} ;
\end{array}\right.
$$

and

$$
R_{2 n+2 m+1}=\ln \left(1+\frac{p_{2 n+2 m+1}^{1}}{1+\sum_{j=1}^{2 m} p_{2 n+j}^{1}}\right)+\ln \left(1+\frac{p_{2 n+2 m+1}^{2}}{1+\sum_{j=1}^{2 m} p_{2 n+j}^{2}}\right)
$$

Moreover, let

$$
\bar{p}_{k}=\left\{\begin{array}{l}
1, \text { if } 1 \leq k \leq 2 n ; \\
4(\sqrt{2}-1), \text { if } 2 n+1 \leq k \leq 2 n+2 m ; \\
2(\sqrt{2}-1)(1+2 n+M+4(\sqrt{2}-1)(m-M)), \text { if } k=2 n+2 m+1 .
\end{array}\right.
$$

Then, the constructed instance of problem (3) is

$$
\begin{array}{ll}
\max _{\left\{p_{k}^{n}\right\}} & \min _{k \in \mathcal{K}}\left\{R_{k}\right\} \\
\text { s.t. } & p_{k}^{1}+p_{k}^{2} \leq 1, k=1,2, \ldots, 2 n, \\
& p_{k}^{1}+p_{k}^{2} \leq 4(\sqrt{2}-1), k=2 n+1,2 n+2, \ldots, 2 n+2 m, \\
& p_{2 n+2 m+1}^{1}+p_{2 n+2 m+1}^{2} \leq 2(\sqrt{2}-1)(1+2 n+M+4(\sqrt{2}-1)(m-M)), \\
& p_{k}^{1} \geq 0, p_{k}^{2} \geq 0, k \in \mathcal{K},
\end{array}
$$

where $R_{k}$ are given in (6), (7), (8), (10), and (11). By using the similar argument as the one in Theorem 1, we can show that the transformation from the MAX-2UNANIMITY problem to problem (13) can be finished in polynomial time. Next, we show that there exists a truth assignment such that at least $M$ clauses are satisfied unanimously for the given MAX-2UNANIMITY instance if and only if the optimal value of problem (13) is greater than or equal to 1 . 
For any $j=1,2, \ldots, m$, since $R_{2 n+j}$ in (8) and $R_{2 n+m+j}$ in (10) are symmetric, it follows that $p_{2 n+j}^{1}=p_{2 n+m+j}^{2}$ and $p_{2 n+m+j}^{1}=p_{2 n+j}^{2}$ at the optimal solutions of problem (13). This shows that

$$
\sum_{j=1}^{2 m} p_{2 n+j}^{1}=\sum_{j=1}^{2 m} p_{2 n+j}^{2}=\sum_{j=1}^{m} p_{2 n+j}^{1}+\sum_{j=1}^{m} p_{2 n+j}^{2}
$$

holds at the optimal solutions of problem (13), which, together with the fact

$$
\bar{p}_{2 n+2 m+1}=2(\sqrt{2}-1)(1+2 n+M+4(\sqrt{2}-1)(m-M)),
$$

implies that the optimal value of problem (13) is greater than or equal to 1 if and only if

$$
\sum_{j=1}^{m} p_{2 n+j}^{1}+\sum_{j=1}^{m} p_{2 n+j}^{2}
$$

at the optimal solution is less than or equal to $2 n+M+4(\sqrt{2}-1)(m-M)$. Furthermore, since the latter is true if and only if there exists a truth assignment such that $M$ clauses in the given MAX-2UNANIMITY instance are unanimous (cf. Theorem 1), we conclude that checking the optimal value of problem (13) is greater than or equal to 1 is strongly NP-hard. Therefore, problem (3) is strongly NP-hard.

Three remarks are in order. First, although Theorems 1 and 2 concentrate on the strong NPhardness of problems (2) and (3) where $N=2$, it is simple to use the same arguments to show the strong NP-hardness of more general problems (2) and (3) where $N \geq 2$. Table [I] summarizes the complexity status of spectrum management problems (2) and (3). Second, although this paper focuses on spectrum management problems (2) and (3), the developed techniques can be applied to show the NP-hardness of other related optimization problems involving "two" arising from signal processing and wireless communications. For instance, the similar techniques have been used in [27] to show the NP-hardness of the harmonic-mean maximization problem under individual power constraints in the multi-user single-subcarrier multi-input single-output interference channel where each transmitter is equipped with two (or more) antennas. In Section III-C, we shall also extend the techniques to show the strong NP-hardness of the linear transceiver design problem in the multi-user single-subcarrier multi-input multi-output (MIMO) interference channel where all transmitters and receivers are equipped with two (or more) antennas. Finally, we were drawn attention to the work [28] after the submission of this paper. In [28], the authors showed that problem (3) is NP-hard when $N=2$ based on a polynomial time reduction from the 
partition problem [29]. In contrast, we show in this paper that problem (3) is strongly NP-hard when $N=2$ based on a polynomial time reduction from the MAX-2UNANIMITY problem. Therefore, our proof technique is different from the one in [28] and our result is more stronger.

TABLE II

Complexity Status of Spectrum Management for Multi-User Multi-SubCarrier Communication Systems

\begin{tabular}{|c|c|c|}
\hline \# of Subcarriers & Total Power Minimization Problem 2 & Min-Rate Maximization Problem 3 \\
\hline$N=1$ & Polynomial Time Solvable [3] & Polynomial Time Solvable [3] \\
\hline$N \geq 2$ & Strongly NP-hard (Theorem 1 ) & Strongly NP-hard (Theorem 2) \\
\hline
\end{tabular}

\section{Complexity Analysis of Linear Transceiver Design Problems}

In this subsection, we first introduce two formulations of the linear transceiver design problem in the multi-user single-carrier MIMO interference channel and then apply our previously developed techniques to show that both of the problems are strongly NP-hard when all transmitters and receivers are equipped with two antennas.

Consider a $K$-user single-carrier MIMO interference channel where the $k$-th transmitter and receiver are equipped with $N_{k}$ and $M_{k}$ antennas, respectively. The received signal at receiver $k$ is

$$
\mathbf{y}_{k}=\mathbf{H}_{k, k} \mathbf{v}_{k} s_{k}+\sum_{j \neq k} \mathbf{H}_{k, j} \mathbf{v}_{j} s_{j}+\mathbf{z}_{k},
$$

where $\mathbf{H}_{k, j} \in \mathbb{C}^{M_{k} \times N_{j}}$ is the channel matrix from transmitter $j$ to receiver $k, \mathbf{v}_{k} \in \mathbb{C}^{N_{k} \times 1}$ is the beamformer used by transmitter $k, s_{k} \in \mathbb{C}$ is the symbol that transmitter $k$ wishes to send to receiver $k$, and $\mathbf{z}_{k} \in \mathbb{C}^{M_{k} \times 1}$ is the additive white Gaussian noise (AWGN) with distribution $\mathcal{C N}\left(\mathbf{0}, \eta_{k} \mathbf{I}\right)$. Each receiver uses a linear receive strategy and let $\mathbf{u}_{k} \in \mathbb{C}^{M_{k} \times 1}$ be the receive beamformer of receiver $k$. Then, the linearly processed signal at the $k$-th receiver is

$$
\hat{s}_{k}=\mathbf{u}_{k}^{\dagger} \mathbf{y}_{k}
$$

where $(\cdot)^{\dagger}$ denotes the Hermitian operator. Treating interference as noise, we can write the SINR of user $k$ as

$$
\operatorname{SINR}_{k}=\frac{\left|\mathbf{u}_{k}^{\dagger} \mathbf{H}_{k, k} \mathbf{v}_{k}\right|^{2}}{\eta_{k}\left\|\mathbf{u}_{k}\right\|^{2}+\sum_{j \neq k}\left|\mathbf{u}_{k}^{\dagger} \mathbf{H}_{k, j} \mathbf{v}_{j}\right|^{2}}
$$


We consider the following two formulations of the linear transceiver design problem:

$$
\begin{array}{cl}
\max _{\left\{\mathbf{u}_{k}, \mathbf{v}_{k}\right\}} & \sum_{k \in \mathcal{K}}\left\|\mathbf{v}_{k}\right\|^{2} \\
\text { s.t. } & \operatorname{SINR}_{k} \geq \gamma_{k},\left\|\mathbf{u}_{k}\right\|^{2}=1, k \in \mathcal{K},
\end{array}
$$

and

$$
\begin{array}{cl}
\max _{\left\{\mathbf{u}_{k}, \mathbf{v}_{k}\right\}} & \min _{k \in \mathcal{K}}\left\{\operatorname{SINR}_{k}\right\} \\
\text { s.t. } & \left\|\mathbf{u}_{k}\right\|^{2}=1,\left\|\mathbf{v}_{k}\right\|^{2} \leq \bar{p}_{k}, k \in \mathcal{K},
\end{array}
$$

where $\gamma_{k}$ is the desired SINR target of user $k$ and $\bar{p}_{k}$ is the power budget of transmitter $k$. Notice that the norm of all receive beamformers is normalized to be one in problems (14) and (15).

We have the following strong NP-hardness results.

Theorem 3: Problem (14) is strongly NP-hard when $M_{k}=N_{k}=2$ for all $k \in \mathcal{K}$.

The detailed proof of Theorem 3 can be found in Appendix C. By using the similar argument in the proof of Theorem 3 and the similar technique as in the proof of Theorem 2, we can show the following Theorem 4 . We leave the proof of Theorem 4 as an exercise for the interested readers.

Theorem 4: Problem (15) is strongly NP-hard when $M_{k}=N_{k}=2$ for all $k \in \mathcal{K}$.

Notice that the same strong NP-hardness results in Theorems 3 and 4 hold true for more general problems (14) and (15) where $\min \left\{M_{k}, N_{k}\right\} \geq 2$ for all $\mathcal{K}$, although Theorems 3 and 4 concentrate on the special case where $M_{k}=N_{k}=2$ for all $\mathcal{K}$. Table III summarizes the complexity status of linear transceiver design problems (14) and (15). Next, we give some remarks on the complexity of max-min fairness linear transceiver design problem (15).

Problem (15) is shown to be strongly NP-hard in [30], [31] when $\min \left\{M_{k}, N_{k}\right\} \geq 2$ and $M_{k}+N_{k} \geq 5$ for all $k \in \mathcal{K}$. The proof in [30], [31] is based on a polynomial time reduction from the 3-SATISFIABILITY problem. Then, (a variant of) problem (15) is shown to remain strongly NP-hard in [32] when $\min \left\{M_{k}, N_{k}\right\} \geq 2$ for all $k \in \mathcal{K}$. The proof in [32] is based on a polynomial time reduction from the same NP-hard problem as the one used in [31]. In this paper, we show the strong NP-hardness of problem (15) when $\min \left\{M_{k}, N_{k}\right\} \geq 2$ for all $k \in \mathcal{K}$ by establishing a polynomial time reduction from the MAX2-UNANIMITY problem, which is different from the ones in [30]-[32]. Moreover, our NP-hardness result is stronger than the one in [32], since the result in [32] holds true only for complex channel matrices while our result holds true for both complex and real channel matrices. 
TABLE III

Complexity Status of Linear Transceiver Design Problems 14 and 15

\begin{tabular}{|c|c|c|}
\hline \hline \# of Rx Antennas & $N_{k}=1$ & $N_{k} \geq 2$ \\
\hline$M_{k}=1$ & Polynomial Time Solvable [3] & Polynomial Time Solvable [27], [33] \\
\hline$M_{k} \geq 2$ & Polynomial Time Solvable [34] & Strongly NP-hard ([32], Theorems 3] and 4] \\
\hline
\end{tabular}

\section{CONClusions}

Dynamic spectrum management in accordance with fast channel fluctuations can significantly improve spectral efficiency of the multi-user multi-carrier communication system. A major challenge associated with spectrum management is to find, for a given channel state, the globally optimal spectrum management strategy to minimize the total transmission power or maximize the system utility. This paper has provided a complete complexity characterization of the spectrum management problem in the multi-user multi-subcarrier communication system. We have shown that both the total power minimization problem and the min-rate maximization problem are strongly NP-hard when the number of subcarriers is two, and thus answered a long-standing open question in the literature. The complexity results suggest that there is no polynomial time algorithms which can solve the general spectrum management problem to global optimality (unless $\mathrm{P}=\mathrm{NP}$ ) and it is more realistic to design efficient algorithms for finding an approximately optimal or locally optimal spectrum management strategy in polynomial time in practice. It is worthwhile pointing out that the developed techniques in this paper can potentially be extended to show the (strong) NP-hardness of other related optimization problems involving "two" arising from signal processing and wireless communications. Our future work is to design efficient approximation algorithms (with guaranteed approximation ratios) for solving the general dynamic spectrum management problem.

\section{ACKNOWLEDGMENT}

The author thanks Professor Zhi-Quan (Tom) Luo of University of Minnesota and The Chinese University of Hong Kong (Shenzhen) and Professor Yu-Hong Dai of Chinese Academy of Sciences for many useful discussions on an early version of this paper. The author also thanks 
the Associate Editor, Professor Osvaldo Simeone, and three anonymous reviewers for their constructive comments, which significantly improved the quality and presentation of the paper.

\section{APPENDIX A}

\section{A LEMMA}

Lemma 2: The points $\left(p_{1}^{1}, p_{1}^{2}, p_{2}^{1}, p_{2}^{2}\right)^{T}=(1,0,0,1)^{T}$ and $\left(p_{1}^{1}, p_{1}^{2}, p_{2}^{1}, p_{2}^{2}\right)^{T}=(0,1,1,0)^{T}$ are the only feasible solutions of

$$
\left\{\begin{array}{l}
\ln \left(1+\frac{p_{1}^{1}}{1+p_{2}^{1}}\right)+\ln \left(1+\frac{p_{1}^{2}}{1+p_{2}^{2}}\right) \geq \ln 2 \\
\ln \left(1+\frac{p_{2}^{1}}{1+p_{1}^{1}}\right)+\ln \left(1+\frac{p_{2}^{2}}{1+p_{1}^{2}}\right) \geq \ln 2 \\
p_{1}^{1}+p_{1}^{2} \leq 1, p_{2}^{1}+p_{2}^{2} \leq 1 \\
p_{1}^{1} \geq 0, p_{1}^{2} \geq 0, p_{2}^{1} \geq 0, p_{2}^{2} \geq 0
\end{array}\right.
$$

Proof of Lemma 2. We first prove that $\left(p_{1}^{1}, p_{1}^{2}, p_{2}^{1}, p_{2}^{2}\right)^{T}=(1,0,0,1)^{T}$ and $\left(p_{1}^{1}, p_{1}^{2}, p_{2}^{1}, p_{2}^{2}\right)^{T}=$ $(0,1,1,0)^{T}$ are the only two optimal solutions of problem

$$
\begin{array}{ll}
\min _{p_{1}^{1}, p_{1}^{2}, p_{2}^{1}, p_{2}^{2}} & p_{1}^{1}+p_{1}^{2}+p_{2}^{1}+p_{2}^{2} \\
\text { s.t. } & \ln \left(1+\frac{p_{1}^{1}}{1+p_{2}^{1}}\right)+\ln \left(1+\frac{p_{1}^{2}}{1+p_{2}^{2}}\right) \geq \ln 2, \\
& \ln \left(1+\frac{p_{2}^{1}}{1+p_{1}^{1}}\right)+\ln \left(1+\frac{p_{2}^{2}}{1+p_{1}^{2}}\right) \geq \ln 2, \\
& p_{1}^{1} \geq 0, p_{1}^{2} \geq 0, p_{2}^{1} \geq 0, p_{2}^{2} \geq 0 .
\end{array}
$$

The two rate constraints in problem (17) can be equivalently rewritten as

$$
\left(1+p_{1}^{1}+p_{2}^{1}\right)\left(1+p_{1}^{2}+p_{2}^{2}\right) \geq 2\left(1+p_{2}^{1}\right)\left(1+p_{2}^{2}\right)
$$

and

$$
\left(1+p_{1}^{1}+p_{2}^{1}\right)\left(1+p_{1}^{2}+p_{2}^{2}\right) \geq 2\left(1+p_{1}^{1}\right)\left(1+p_{1}^{2}\right)
$$

Adding the above two inequalities together yields $p_{1}^{1} p_{2}^{2}+p_{1}^{2} p_{2}^{1} \geq 1$, which implies that problem

$$
\begin{array}{cl}
\min _{p_{1}^{1}, p_{1}^{2}, p_{2}^{1}, p_{2}^{2}} & p_{1}^{1}+p_{1}^{2}+p_{2}^{1}+p_{2}^{2} \\
\text { s.t. } & p_{1}^{1} p_{2}^{2}+p_{1}^{2} p_{2}^{1} \geq 1, \\
& p_{1}^{1} \geq 0, p_{1}^{2} \geq 0, p_{2}^{1} \geq 0, p_{2}^{2} \geq 0,
\end{array}
$$


is a relaxation of problem (17). If we can show $\left(p_{1}^{1}, p_{1}^{2}, p_{2}^{1}, p_{2}^{2}\right)^{T}=(1,0,0,1)^{T}$ and $\left(p_{1}^{1}, p_{1}^{2}, p_{2}^{1}, p_{2}^{2}\right)^{T}=$ $(0,1,1,0)^{T}$ are the only two optimal solutions of problem $(\mathbf{1 8})$, then they must be the only two optimal solutions of problem (17). This further implies that $\left(p_{1}^{1}, p_{1}^{2}, p_{2}^{1}, p_{2}^{2}\right)^{T}=(1,0,0,1)^{T}$ and $\left(p_{1}^{1}, p_{1}^{2}, p_{2}^{1}, p_{2}^{2}\right)^{T}=(0,1,1,0)^{T}$ are the only feasible points of

$$
\left\{\begin{array}{l}
\ln \left(1+\frac{p_{1}^{1}}{1+p_{2}^{1}}\right)+\ln \left(1+\frac{p_{1}^{2}}{1+p_{2}^{2}}\right) \geq \ln 2, \\
\ln \left(1+\frac{p_{2}^{1}}{1+p_{1}^{1}}\right)+\ln \left(1+\frac{p_{2}^{2}}{1+p_{1}^{2}}\right) \geq \ln 2, \\
p_{1}^{1}+p_{1}^{2}+p_{2}^{1}+p_{2}^{2} \leq 2, \\
p_{1}^{1} \geq 0, p_{1}^{2} \geq 0, p_{2}^{1} \geq 0, p_{2}^{2} \geq 0 .
\end{array}\right.
$$

Since

$\left\{\left(p_{1}^{1}, p_{1}^{2}, p_{2}^{1}, p_{2}^{2}\right) \geq 0 \mid p_{1}^{1}+p_{1}^{2} \leq 1, p_{2}^{1}+p_{2}^{2} \leq 1\right\} \subseteq\left\{\left(p_{1}^{1}, p_{1}^{2}, p_{2}^{1}, p_{2}^{2}\right) \geq 0 \mid p_{1}^{1}+p_{1}^{2}+p_{2}^{1}+p_{2}^{2} \leq 2\right\} ，$ it follows that $\left(p_{1}^{1}, p_{1}^{2}, p_{2}^{1}, p_{2}^{2}\right)^{T}=(1,0,0,1)^{T}$ and $\left(p_{1}^{1}, p_{1}^{2}, p_{2}^{1}, p_{2}^{2}\right)^{T}=(0,1,1,0)^{T}$ are the only feasible points of (16).

It remains to prove that $\left(p_{1}^{1}, p_{1}^{2}, p_{2}^{1}, p_{2}^{2}\right)^{T}=(1,0,0,1)^{T}$ and $\left(p_{1}^{1}, p_{1}^{2}, p_{2}^{1}, p_{2}^{2}\right)^{T}=(0,1,1,0)^{T}$ are the only two optimal solutions of problem (18). It can be verified that the optimal solution of the following problem

$$
\begin{array}{cl}
\min _{p_{1}^{1}, p_{1}^{2}, p_{2}^{1}, p_{2}^{2}} & 2\left(\sqrt{p_{1}^{1} p_{2}^{2}}+\sqrt{p_{1}^{2} p_{2}^{1}}\right) \\
\text { s.t. } & p_{1}^{1} p_{2}^{2}+p_{1}^{2} p_{2}^{1} \geq 1, \\
& p_{1}^{1} \geq 0, p_{1}^{2} \geq 0, p_{2}^{1} \geq 0, p_{2}^{2} \geq 0,
\end{array}
$$

must satisfy

$$
p_{1}^{1} p_{2}^{2}=1, p_{1}^{2} p_{2}^{1}=0
$$

or

$$
p_{1}^{1} p_{2}^{2}=0, p_{1}^{2} p_{2}^{1}=1,
$$

and its optimal value is 2 . Since $p_{1}^{1}+p_{2}^{2} \geq 2 \sqrt{p_{1}^{1} p_{2}^{2}}$ and $p_{1}^{2}+p_{2}^{1} \geq 2 \sqrt{p_{1}^{2} p_{2}^{1}}$ and the above two inequalities hold true with " $=$ " if and only if $p_{1}^{1}=p_{2}^{2}$ and $p_{1}^{2}=p_{2}^{1}$, we conclude that the only points that achieve the objective value of problem (18) of being 2 are $\left(p_{1}^{1}, p_{1}^{2}, p_{2}^{1}, p_{2}^{2}\right)^{T}=(1,0,0,1)^{T}$ and $\left(p_{1}^{1}, p_{1}^{2}, p_{2}^{1}, p_{2}^{2}\right)^{T}=(0,1,1,0)^{T}$. Hence, $\left(p_{1}^{1}, p_{1}^{2}, p_{2}^{1}, p_{2}^{2}\right)^{T}=(1,0,0,1)^{T}$ and $\left(p_{1}^{1}, p_{1}^{2}, p_{2}^{1}, p_{2}^{2}\right)^{T}=$ $(0,1,1,0)^{T}$ are the only two optimal solutions of problem (18). This completes the proof of Lemma 2 . 


\section{APPENDIX B}

\section{AN ILLUSTRATIVE EXAMPLE}

Consider the instance in Example 1. Then there are 12 users in the constructed 2-carrier communication system, including 4 "variable user" (denoted as user 1,2,3,4), 4 "auxiliary variable user" (denoted as user 5,6,7,8), and 4 "clause user" (denoted as user 9, 10,11, 12). In this case, $\mathcal{K}=\{1,2, \ldots, 12\}$, and all users' rate expressions are given as follows:

$$
\begin{aligned}
& R_{1}=\ln \left(1+\frac{p_{1}^{1}}{1+p_{5}^{1}}\right)+\ln \left(1+\frac{p_{1}^{2}}{1+p_{5}^{2}}\right), \\
& R_{2}=\ln \left(1+\frac{p_{2}^{1}}{1+p_{6}^{1}}\right)+\ln \left(1+\frac{p_{2}^{2}}{1+p_{6}^{2}}\right), \\
& R_{3}=\ln \left(1+\frac{p_{3}^{1}}{1+p_{7}^{1}}\right)+\ln \left(1+\frac{p_{3}^{2}}{1+p_{7}^{2}}\right), \\
& R_{4}=\ln \left(1+\frac{p_{4}^{1}}{1+p_{8}^{1}}\right)+\ln \left(1+\frac{p_{4}^{2}}{1+p_{8}^{2}}\right), \\
& R_{5}=\ln \left(1+\frac{p_{5}^{1}}{1+p_{1}^{1}}\right)+\ln \left(1+\frac{p_{5}^{2}}{1+p_{1}^{2}}\right) \text {, } \\
& R_{6}=\ln \left(1+\frac{p_{6}^{1}}{1+p_{2}^{1}}\right)+\ln \left(1+\frac{p_{6}^{2}}{1+p_{2}^{2}}\right), \\
& R_{7}=\ln \left(1+\frac{p_{7}^{1}}{1+p_{3}^{1}}\right)+\ln \left(1+\frac{p_{7}^{2}}{1+p_{3}^{2}}\right), \\
& R_{8}=\ln \left(1+\frac{p_{8}^{1}}{1+p_{4}^{1}}\right)+\ln \left(1+\frac{p_{8}^{2}}{1+p_{4}^{2}}\right), \\
& R_{9}=\ln \left(1+\frac{p_{9}^{1}}{1+p_{1}^{1}+p_{6}^{1}}\right)+\ln \left(1+\frac{p_{9}^{2}}{1+p_{1}^{2}+p_{6}^{2}}\right) \text {, } \\
& R_{10}=\ln \left(1+\frac{p_{10}^{1}}{1+p_{1}^{1}+p_{3}^{1}}\right)+\ln \left(1+\frac{p_{10}^{2}}{1+p_{1}^{2}+p_{3}^{2}}\right) \text {, } \\
& R_{11}=\ln \left(1+\frac{p_{11}^{1}}{1+p_{6}^{1}+p_{8}^{1}}\right)+\ln \left(1+\frac{p_{11}^{2}}{1+p_{6}^{2}+p_{8}^{2}}\right) \text {, } \\
& R_{12}=\ln \left(1+\frac{p_{12}^{1}}{1+p_{7}^{1}+p_{4}^{1}}\right)+\ln \left(1+\frac{p_{12}^{2}}{1+p_{7}^{2}+p_{4}^{2}}\right) \text {. }
\end{aligned}
$$




\section{APPENDIX C \\ PROOF OF THEOREM 3}

To ease the presentation, we first define some notation. Let

$$
\begin{gathered}
\mathbf{e}_{1}=\left(\begin{array}{l}
1 \\
0
\end{array}\right), \mathbf{e}_{2}=\left(\begin{array}{l}
0 \\
1
\end{array}\right), \mathbf{e}=\left(\begin{array}{l}
1 \\
1
\end{array}\right), \\
\mathbf{H}_{A}=\left(\begin{array}{ll}
1 & 0 \\
0 & 1
\end{array}\right), \mathbf{H}_{B}=\left(\begin{array}{ll}
0 & 1 \\
1 & 0
\end{array}\right), \mathbf{H}_{C}=\left(\begin{array}{ll}
0 & 1 \\
0 & 0
\end{array}\right) .
\end{gathered}
$$

For any given two vectors $\mathbf{u}$ and $\mathbf{v}$ with $\|\mathbf{u}\|=\|\mathbf{v}\|=1$, we denote $\mathbf{u} \simeq \mathbf{v}$ if there exists a scaler $r \in \mathbb{C}$ (with $|r|=1$ ) such that $\mathbf{u}=r \mathbf{v}$.

To show Theorem 3, we need the following lemma, which recognizes a discrete structure in the solution of a special instance of the decision version of problem (14).

Lemma 3: Consider the following problem instance

$$
\left\{\begin{array}{l}
\left|\mathbf{u}_{1}^{\dagger} \mathbf{H}_{A} \mathbf{v}_{1}\right|^{2} \geq 1+\left|\mathbf{u}_{1}^{\dagger} \mathbf{H}_{B} \mathbf{v}_{2}\right|^{2}+\left|\mathbf{u}_{1}^{\dagger} \mathbf{H}_{B} \mathbf{v}_{3}\right|^{2} \\
\left|\mathbf{u}_{2}^{\dagger} \mathbf{H}_{A} \mathbf{v}_{2}\right|^{2} \geq 1+\left|\mathbf{u}_{2}^{\dagger} \mathbf{H}_{C} \mathbf{v}_{3}\right|^{2} \\
\left|\mathbf{u}_{3}^{\dagger} \mathbf{H}_{A} \mathbf{v}_{3}\right|^{2} \geq 1+\left|\mathbf{u}_{3}^{\dagger} \mathbf{H}_{C} \mathbf{v}_{2}\right|^{2} \\
\left\|\mathbf{v}_{1}\right\|^{2}+\left\|\mathbf{v}_{2}\right\|^{2}+\left\|\mathbf{v}_{3}\right\|^{2} \leq 3 \\
\left\|\mathbf{u}_{1}\right\|^{2}=1,\left\|\mathbf{u}_{2}\right\|^{2}=1,\left\|\mathbf{u}_{3}\right\|^{2}=1
\end{array}\right.
$$

The points $\mathbf{u}_{1} \simeq \mathbf{u}_{2} \simeq \mathbf{u}_{3} \simeq \mathbf{v}_{1} \simeq \mathbf{v}_{2} \simeq \mathbf{v}_{3} \simeq \mathbf{e}_{1}$ and $\mathbf{u}_{1} \simeq \mathbf{u}_{2} \simeq \mathbf{u}_{3} \simeq \mathbf{v}_{1} \simeq \mathbf{v}_{2} \simeq \mathbf{v}_{3} \simeq \mathbf{e}_{2}$ are the only two feasible solutions of problem (20) (up to an arbitrary phase rotation).

Proof of Lemma 3. We first prove that the necessary conditions for problem (20) being feasible are

$$
\begin{gathered}
\left\|\mathbf{v}_{1}\right\|=\left\|\mathbf{v}_{2}\right\|=\left\|\mathbf{v}_{3}\right\|=1 \\
\mathbf{u}_{1} \simeq \mathbf{v}_{1}, \quad \mathbf{u}_{2} \simeq \mathbf{v}_{2}, \quad \mathbf{u}_{3} \simeq \mathbf{v}_{3},
\end{gathered}
$$

and

$$
\mathbf{u}_{1}^{\dagger} \mathbf{H}_{B} \mathbf{v}_{2}=0, \mathbf{u}_{1}^{\dagger} \mathbf{H}_{B} \mathbf{v}_{3}=0, \mathbf{u}_{2}^{\dagger} \mathbf{H}_{C} \mathbf{v}_{3}=0
$$

We prove (21), (22), and (23) sequentially. 
Proof of 21): We prove (21) based on the contradiction principle. We divide the proof into two cases: Case 1 (there exists an $i=1,2,3$ such that $\left\|\mathbf{v}_{i}\right\|<1$ ) and Case 2 (there exists an $i=1,2,3$ such that $\left.\left\|\mathbf{v}_{i}\right\|>1\right)$.

- Case 1: Without loss of generality, suppose that $\left\|\mathbf{v}_{1}\right\|<1$. This, together with the fact $\left\|\mathbf{u}_{1}\right\|=1$, implies $\left|\mathbf{u}_{1}^{\dagger} \mathbf{H}_{A} \mathbf{v}_{1}\right|^{2}<1$, which contradicts the first equation of (20). Consequently, we must have $\left\|\mathbf{v}_{i}\right\| \geq 1$ for all $i=1,2,3$. Combining this with the fourth equation of (20), we immediately obtain (21).

- Case 2: Without loss of generality, suppose that $\left\|\mathbf{v}_{1}\right\|>1$. Then, by the fourth equation of (20), we must have $\left\|\mathbf{v}_{2}\right\|<1$ or $\left\|\mathbf{v}_{3}\right\|<1$. This reduces to Case 1 and therefore (21) is true.

Proof of (22): It follows from the first three conditions of (20) that $\left|\mathbf{u}_{i}^{\dagger} \mathbf{v}_{i}\right| \geq 1$ for all $i=1,2,3$. This and the facts $\left\|\mathbf{u}_{i}\right\|=\left\|\mathbf{v}_{i}\right\|=1$ for all $i=1,2,3$ imply (22).

Proof of (23): By (21), (22), and the last condition of (20), we have $\left|\mathbf{u}_{i}^{\dagger} \mathbf{H}_{A} \mathbf{v}_{i}\right|=1$ for all $i=1,2,3$. This, together with the first three conditions in (20), immediately implies (23).

Next, we show the truth of the lemma based on (21), (22), and (23). We focus on showing that $\mathbf{v}_{1} \simeq \mathbf{e}_{1}$ or $\mathbf{v}_{1} \simeq \mathbf{e}_{2}$. Based on this, we can immediately obtain the other results in the lemma by using (22) and (23). Let

$$
\mathbf{v}_{1}=\left(\begin{array}{l}
v_{11} \\
v_{12}
\end{array}\right)
$$

with $\left\|\mathbf{v}_{1}\right\|=1$. It suffices to prove that either $v_{11}=0$ or $v_{12}=0$. Combining (22) and (23) yields

$$
\mathbf{v}_{1}^{\dagger} \mathbf{H}_{B} \mathbf{v}_{2}=0, \mathbf{v}_{1}^{\dagger} \mathbf{H}_{B} \mathbf{v}_{3}=0, \mathbf{v}_{2}^{\dagger} \mathbf{H}_{C} \mathbf{v}_{3}=0 .
$$

By the first two conditions of (24), we obtain

$$
\mathbf{v}_{2} \simeq \mathbf{H}_{B}\left(\begin{array}{c}
\bar{v}_{12} \\
-\bar{v}_{11}
\end{array}\right) \text { and } \mathbf{v}_{3} \simeq \mathbf{H}_{B}\left(\begin{array}{c}
\bar{v}_{12} \\
-\bar{v}_{11}
\end{array}\right),
$$

where $\bar{v}$ denotes the conjugate of $v \in \mathbb{C}$. Substituting (25) into the third condition of (24), we get

$$
\left(\begin{array}{c}
\bar{v}_{12} \\
-\bar{v}_{11}
\end{array}\right)^{\dagger} \mathbf{H}_{B} \mathbf{H}_{C} \mathbf{H}_{B}\left(\begin{array}{c}
\bar{v}_{12} \\
-\bar{v}_{11}
\end{array}\right)=0,
$$


which is equivalent to

$$
v_{11} \bar{v}_{12}=0 .
$$

This shows that either $v_{11}$ or $v_{12}$ is zero. Therefore, we get either $\mathbf{v}_{1} \simeq \mathbf{e}_{1}$ or $\mathbf{v}_{1} \simeq \mathbf{e}_{2}$. The proof of Lemma 3 is completed.

We are now ready to prove Theorem 3 ,

Proof of Theorem [3: Given any instance of the MAX-2UNANIMITY problem with clauses $c_{1}, c_{2}, \ldots, c_{m}$ defined over Boolean variables $x_{1}, x_{2}, \ldots, x_{n}$ and an integer $M$, we construct below a multi-user MIMO interference channel with $3 n+m$ users, where the Boolean variable $x_{i}(i=1,2, \ldots, n)$ corresponds to three users, including users $i, n+i$, and $2 n+i$; each clause $c_{j}(j=1,2, \ldots, m)$ corresponds to user $3 n+j$. Hence, $\mathcal{K}=\{1,2, \ldots, 3 n+m\}$.

We now construct the direct-link and crosstalk channel matrices for all $3 n+m$ users. All the direct-link channel matrices are set to be

$$
\mathbf{H}_{k, k}=\mathbf{H}_{A}, k \in \mathcal{K} .
$$

The corresponding crosstalk channel matrices are: for user $k, k=1,2, \ldots, n$, set

$$
\mathbf{H}_{k, n+k}=\mathbf{H}_{k, 2 n+k}=\mathbf{H}_{B} \text { and } \mathbf{H}_{k, j}=\mathbf{0}, \forall k \in \mathcal{K} \backslash\{k, n+k, 2 n+k\} ;
$$

for user $k, k=n+1, n+2, \ldots, 2 n$, set

$$
\mathbf{H}_{k, n+k}=\mathbf{H}_{C} \text { and } \mathbf{H}_{k, j}=\mathbf{0}, \forall k \in \mathcal{K} \backslash\{k, n+k\} ;
$$

for user $k, k=2 n+1,2 n+2, \ldots, 3 n$, set

$$
\mathbf{H}_{k, k-n}=\mathbf{H}_{C} \text { and } \mathbf{H}_{k, j}=\mathbf{0}, \forall k \in \mathcal{K} \backslash\{k-n, k\} ;
$$

and for user $k, k=3 n+1,3 n+2, \ldots, 3 n+m, \mathbf{H}_{k, j}=\mathbf{0}$ for all $\mathcal{K} \backslash\{k\}$ except

$$
\begin{aligned}
& \mathbf{H}_{k, j}= \begin{cases}\mathbf{H}_{A}, & \text { if } \alpha_{\pi(k)}=x_{j} \text { for some } j ; \\
\mathbf{H}_{B}, & \text { if } \alpha_{\pi(k)}=\bar{x}_{j} \text { for some } j,\end{cases} \\
& \mathbf{H}_{k, j}= \begin{cases}\mathbf{H}_{A}, & \text { if } \beta_{\rho(k)}=x_{j} \text { for some } j ; \\
\mathbf{H}_{B}, & \text { if } \beta_{\rho(k)}=\bar{x}_{j} \text { for some } j,\end{cases}
\end{aligned}
$$

where $c_{k-3 n}=\alpha_{\pi(k)} \vee \beta_{\rho(k)}, \alpha$ and $\beta$ are taken from $\{x, \bar{x}\}$, and $\pi$ and $\rho$ are mappings from $\{3 n+1,3 n+2, \ldots, 3 n+m\}$ to $\{1,2, \ldots, n\}$. Set $\eta_{k}=1$ for all $k \in \mathcal{K}$. Then, the SINR expressions 
of all users are: for $i=1,2, \ldots, n$,

$$
\begin{gathered}
\operatorname{SINR}_{i}=\frac{\left|\mathbf{u}_{i}^{\dagger} \mathbf{H}_{A} \mathbf{v}_{i}\right|^{2}}{1+\left|\mathbf{u}_{i}^{\dagger} \mathbf{H}_{B} \mathbf{v}_{n+i}\right|^{2}+\left|\mathbf{u}_{i}^{\dagger} \mathbf{H}_{B} \mathbf{v}_{2 n+i}\right|^{2}} \\
\operatorname{SINR}_{n+i}=\frac{\left|\mathbf{u}_{n+i}^{\dagger} \mathbf{H}_{A} \mathbf{v}_{n+i}\right|^{2}}{1+\left|\mathbf{u}_{n+i}^{\dagger} \mathbf{H}_{C} \mathbf{v}_{2 n+i}\right|^{2}}
\end{gathered}
$$

and

$$
\operatorname{SINR}_{2 n+i}=\frac{\left|\mathbf{u}_{2 n+i}^{\dagger} \mathbf{H}_{A} \mathbf{v}_{2 n+i}\right|^{2}}{1+\left|\mathbf{u}_{2 n+i}^{\dagger} \mathbf{H}_{C} \mathbf{v}_{n+i}\right|^{2}}
$$

for $j=1,2, \ldots, m$,

$$
\mathrm{SINR}_{3 n+j}=\left\{\begin{array}{l}
\frac{\left|\mathbf{u}_{3 n+j}^{\dagger} \mathbf{H}_{A} \mathbf{v}_{3 n+j}\right|^{2}}{1+\left|\mathbf{u}_{3 n+j}^{\dagger} \mathbf{H}_{A} \mathbf{v}_{i_{1}}\right|^{2}+\left|\mathbf{u}_{3 n+j}^{\dagger} \mathbf{H}_{A} \mathbf{v}_{i_{2}}\right|^{2}}, \text { if } c_{j}=x_{i_{1}} \vee x_{i_{2}} \\
\frac{\left|\mathbf{u}_{3 n+j}^{\dagger} \mathbf{H}_{A} \mathbf{v}_{3 n+j}\right|^{2}}{1+\left|\mathbf{u}_{3 n+j}^{\dagger} \mathbf{H}_{A} \mathbf{v}_{i_{1}}\right|^{2}+\left|\mathbf{u}_{3 n+j}^{\dagger} \mathbf{H}_{B} \mathbf{v}_{i_{2}}\right|^{2}}, \text { if } c_{j}=x_{i_{1}} \vee \bar{x}_{i_{2}} \\
\frac{\left|\mathbf{u}_{3 n+j}^{\dagger} \mathbf{H}_{A} \mathbf{v}_{3 n+j}\right|^{2}}{1+\left|\mathbf{u}_{3 n+j}^{\dagger} \mathbf{H}_{B} \mathbf{v}_{i_{1}}\right|^{2}+\left|\mathbf{u}_{3 n+j}^{\dagger} \mathbf{H}_{A} \mathbf{v}_{i_{2}}\right|^{2}}, \text { if } c_{j}=\bar{x}_{i_{1}} \vee x_{i_{2}} \\
\frac{\left|\mathbf{u}_{3 n+j}^{\dagger} \mathbf{H}_{A} \mathbf{v}_{3 n+j}\right|^{2}}{1+\left|\mathbf{u}_{3 n+j}^{\dagger} \mathbf{H}_{B} \mathbf{v}_{i_{1}}\right|^{2}+\left|\mathbf{u}_{3 n+j}^{\dagger} \mathbf{H}_{B} \mathbf{v}_{i_{2}}\right|^{2}}, \text { if } c_{j}=\bar{x}_{i_{1}} \vee \bar{x}_{i_{2}}
\end{array}\right.
$$

Moreover, let $\gamma_{k}=1$ for all $k \in \mathcal{K}$. Then, the constructed instance of problem (14) is

$$
\begin{array}{ll}
\min _{\left\{\mathbf{u}_{k}, \mathbf{v}_{k}\right\}} & \sum_{k \in \mathcal{K}}\left\|\mathbf{v}_{k}\right\|^{2} \\
\text { s.t. } & \operatorname{SINR}_{k} \geq 1, k \in \mathcal{K}, \\
& \left\|\mathbf{u}_{k}\right\|^{2}=1, k \in \mathcal{K},
\end{array}
$$

where $\mathrm{SINR}_{k}$ are given in (27), (28), (29), and (30).

Next, we show that there exists a truth assignment such that at least $M$ clauses are satisfied unanimously for the given MAX-2UNANIMITY instance if and only if the optimal value of problem (31) is less than or equal to $3 n+2 m-M$. 
If there exists a truth assignment such that $M$ clauses in the MAX-2UNANIMITY problem are unanimous, we claim that the optimal value of problem (31) is less than or equal to $3 n+2 m-M$. Let $\left\{x_{i}\right\}$ be the truth assignment such that $M$ clauses are unanimous in the MAX-2UNANIMITY problem. We set

$$
\mathbf{u}_{i}=\mathbf{u}_{n+i}=\mathbf{u}_{2 n+i}=\mathbf{v}_{i}=\mathbf{v}_{n+i}=\mathbf{v}_{2 n+i}=\left(\begin{array}{c}
x_{i} \\
1-x_{i}
\end{array}\right), i=1,2, \ldots, n .
$$

With this, we can simply check that $\operatorname{SINR}_{k} \geq 1$ for all $k=1,2, \ldots, 3 n$. Furthermore, we consider SINR requirements of user $3 n+j$ with $j=1,2, \ldots, m$.

- If the clause $c_{j}$ is unanimous, then we have either

or

$$
\operatorname{SINR}_{3 n+j}=\frac{\left|\mathbf{u}_{3 n+j}^{\dagger} \mathbf{H}_{A} \mathbf{v}_{3 n+j}\right|^{2}}{1+2\left|\mathbf{u}_{3 n+j}^{\dagger} \mathbf{e}_{1}\right|^{2}}
$$

$$
\operatorname{SINR}_{3 n+j}=\frac{\left|\mathbf{u}_{3 n+j}^{\dagger} \mathbf{H}_{A} \mathbf{v}_{3 n+j}\right|^{2}}{1+2\left|\mathbf{u}_{3 n+j}^{\dagger} \mathbf{e}_{2}\right|^{2}}
$$

In either cases, we can use a total transmission power of 1 to make $\operatorname{SINR}_{3 n+j} \geq 1$ satisfied (by setting $\mathbf{u}_{3 n+j}=\mathbf{v}_{3 n+j}=\mathbf{e}_{2}$ in the former case and $\mathbf{u}_{3 n+j}=\mathbf{v}_{3 n+j}=\mathbf{e}_{1}$ in the latter case).

- If the clause $c_{j}$ is not unanimous, then we must have

$$
\operatorname{SINR}_{3 n+j}=\frac{\left|\mathbf{u}_{3 n+j}^{\dagger} \mathbf{H}_{A} \mathbf{v}_{3 n+j}\right|^{2}}{1+\left|\mathbf{u}_{3 n+j}^{\dagger} \mathbf{e}_{1}\right|^{2}+\left|\mathbf{u}_{3 n+j}^{\dagger} \mathbf{e}_{2}\right|^{2}}
$$

In this case, we can use a total transmission power of 2 to make $\operatorname{SINR}_{3 n+j} \geq 1$ satisfied (by setting $\mathbf{u}=\sqrt{2} \mathbf{e} / 2$ and $\mathbf{v}_{3 n+j}=\mathbf{e}$ ).

As a result, if there exists a truth assignment such that at least $M$ clauses are satisfied unanimously, then the optimal value of problem (31) is less than or equal to $3 n+2 m-M$.

For the converse part, assuming that the optimal value of problem (31) is less than or equal to $3 n+2 m-M$, we claim that at least $M$ clauses can be made unanimous. It follows from

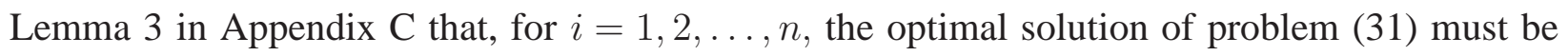

$$
\mathbf{v}_{i} \simeq \mathbf{e}_{1} \text { or } \mathbf{v}_{i} \simeq \mathbf{e}_{2} .
$$


This, together with (30), implies that the interference terms at user $3 n+j$ must be $\left|\mathbf{u}_{3 n+j}^{\dagger} \mathbf{e}_{1}\right|^{2}$ and/or $\left|\mathbf{u}_{3 n+j}^{\dagger} \mathbf{e}_{2}\right|^{2}$ for all $j=1,2, \ldots, m$. More specifically, there might be two cases:

- Case 1: the two interference terms at user $3 n+j$ are the same, i.e., either both of them are $\left|\mathbf{u}_{3 n+j}^{\dagger} \mathbf{e}_{1}\right|^{2}$ or both of them are $\left|\mathbf{u}_{3 n+j}^{\dagger} \mathbf{e}_{2}\right|^{2}$;

- Case 2: the two interference terms at user $3 n+j$ are different, i.e., one is $\left|\mathbf{u}_{3 n+j}^{\dagger} \mathbf{e}_{1}\right|^{2}$ and the other is $\left|\mathbf{u}_{3 n+j}^{\dagger} \mathbf{e}_{2}\right|^{2}$.

If Case 1 happens for user $3 n+j$, then the required total transmission power satisfying $\operatorname{SINR}_{3 n+j} \geq$ 1 is at least 1 ; while if Case 2 happens for user $3 n+j$, then the required total transmission power satisfying $\operatorname{SINR}_{3 n+j} \geq 1$ is at least 2 . By the assumption that the optimal value of problem (31) is less than or equal to $3 n+2 m-M$, we know that Case 1 must happen at least $M$ times (Case 2 cannot happen more than $m-M$ times). Moreover, it can be checked that

$$
x_{i}=\left|v_{i 1}\right| \in\{0,1\}, i=1,2, \ldots, n
$$

is a truth assignment which makes at least $M$ clauses in the MAX-2UNANIMITY problem satisfied unanimuously, where $v_{i 1}$ is the first component of the vector $\mathbf{v}_{i}$.

Finally, this transformation is in polynomial time. Since the MAX-2UNANIMITY problem is NP-complete (cf. Lemma 1), we conclude that the problem of checking the optimal value of problem (31) is less than or equal to $3 n+2 m-M$ is strongly NP-hard. Hence, problem (14) is strongly NP-hard. The proof of Theorem 3 is completed.

\section{REFERENCES}

[1] A. Goldsmith, Wireless communications. Cambridge university press, 2005.

[2] D. Tse and P. Viswanath, Fundamentals of wireless communication. Cambridge university press, 2005.

[3] Z.-Q. Luo and S. Zhang, "Dynamic spectrum management: Complexity and duality," J. Sel. Topics Signal Process., vol. 2, no. 1, pp. 57-73, Feb. 2008.

[4] S. Hayashi and Z.-Q. Luo, "Spectrum management for interference-limited multiuser communication systems," IEEE Trans. Inf. Theory, vol. 55, no. 3, pp. 1153-1175, Mar. 2009.

[5] Y.-F. Liu and Y.-H. Dai, "On the complexity of joint subcarrier and power allocation for multi-user OFDMA systems," IEEE Trans. Signal Process., vol. 62, no. 3, pp. 583-596, Feb. 2014.

[6] W. Yu, G. Ginis, and J. M. Cioffi, "Distributed multiuser power control for digital subscriber lines," IEEE J. Sel. Areas Commun., vol. 20, no. 5, pp. 1105-1115, June 2002.

[7] Z.-Q. Luo and J.-S. Pang, "Analysis of iterative waterfilling algorithm for multiuser power control in digital subscriber lines," EURASIP J. Adv. Siginal Process., vol. 2006, pp. 1-10, Mar. 2006. 
[8] R. Cendrillon, W. Yu, M. Moonen, J. Verlinden, and T. Bostoen, "Optimal multiuser spectrum balancing for digital subscriber lines," IEEE Trans. Commun., vol. 54, no. 5, pp. 922-933, May 2006.

[9] R. Cendrillon and M. Moonen, "Iterative spectrum balancing for digital subscriber lines," in Proc. IEEE Int. Conf. Commun., Seoul, Korea, 16-20 May 2005, pp. 1937-1941.

[10] R. Lui and W. Yu, "Low-complexity near-optimal spectrum balancing for digital subscriber lines," in Proc. IEEE Int. Conf. Commun., Seoul, Korea, 16-20 May 2005, pp. 1947-1951.

[11] W. Yu and R. Lui, "Dual methods for nonconvex spectrum optimization of multicarrier systems," IEEE Trans. Commun., vol. 54, no. 7, pp. 1310-1322, July 2006.

[12] J. Papandriopoulos and J. S. Evans, "SCALE: a low-complexity distributed protocol for spectrum balancing in multiuser DSL networks," IEEE Trans. Inf. Theory, vol. 55, no. 8, pp. 3711-3724, Aug. 2009.

[13] Z.-Q. Luo and S. Zhang, "Duality gap estimation and polynomial time approximation for optimal spectrum management," IEEE Trans. Signal Process., vol. 57, no. 7, pp. 2675-2689, July 2009.

[14] T. Wang and L. Vandendorpe, "On the SCALE algorithm for multiuser multicarrier power spectrum management," IEEE Trans. Signal Process., vol. 60, no. 9, pp. 4992-4998, Sept. 2012.

[15] P. Tsiaflakis, F. Glineur, and M. Moonen, "Real-time dynamic spectrum management for multi-user multi-carrier communication systems," IEEE Trans. Commun., vol. 62, no. 3, pp. 1124-1137, Mar. 2014.

[16] S. Sharma and O. P. Sahu, "A modified low complexity based distributed iterative water-filling (IWF) spectrum management algorithm," Wireless Pers. Commun., vol. 82, no. 3, pp. 1239-1247, June 2015.

[17] P. Tsiaflakis, I. Necoara, J. A. Suykens, and M. Moonen, "Improved dual decomposition based optimization for DSL dynamic spectrum management," IEEE Trans. Signal Process., vol. 58, no. 4, pp. 2230-2245, Apr. 2010.

[18] R. B. Moraes, P. Tsiaflakis, J. Maes, and M. Moonen, "General framework and algorithm for data rate maximization in DSL networks," IEEE Trans. Commun., vol. 62, no. 5, pp. 1691-1703, May 2014.

[19] Y. Liu, "Complexity analysis of joint subcarrier and power allocation for the cellular downlink OFDMA system," Wireless Commun. Lett., 2014.

[20] W. Yu, "Multiuser water-filling in the presence of crosstalk," in Proc. Inf. Theory Appl. Workshop, 29 Jan.-2 Feb. 2007, La Jolla, CA, pp. 414-420.

[21] R. B. Moraes, M. Wolkerstorfer, P. Tsiaflakis, and M. Moonen, "Dynamic spectrum management with spherical coordinates," IEEE Trans. Signal Process., vol. 62, no. 21, pp. 5589-5602, Nov. 2014.

[22] P. Liu, Y.-F. Liu, and J. Li, "An iterative reweighted minimization framework for joint channel and power allocation in the OFDMA system," in Proc. IEEE Int. Conf. Acoust. Speech and Signal Process. (ICASSP), Apr. 2015, pp. 3068-3072.

[23] M. R. Garey and D. S. Johnson, Computers and Intractability: A Guide to the Theory of NP-Completeness. W. H. Freeman and Company, 1979.

[24] C. H. Papadimitriou, Computational Complexity. Addison-Wesley, 1994.

[25] V. V. Vazirani, Approximation Algorithms. Springer-Verlag, 2001.

[26] C. H. Papadimitriou and K. Steiglitz, Combinatorial Optimization: Algorithms and Complexity. Prentice-Hall, Inc., 1998.

[27] Y.-F. Liu, Y.-H. Dai, and Z.-Q. Luo, "Coordinated beamforming for MISO interference channel: Complexity analysis and efficient algorithms," IEEE Trans. Signal Process., vol. 59, no. 3, pp. 1142-1157, Mar. 2011.

[28] M. Locatelli and Z.-Q. Luo, "On an open question about the complexity of a dynamic spectrum management problem," Tech. Report, Dec. 2014. 
[29] M. R. Garey and D. S. Johnson, Computers and Intractability: A Guide to the Theory of NP-Completeness. New York: W. H. Freeman, 1979.

[30] Y.-F. Liu, Y.-H. Dai, and Z.-Q. Luo, "Max-min fairness linear transceiver design for a multi-user MIMO interference channel," in Proc. IEEE Int. Conf. Commun. (ICC), Jun. 2011, pp. 1-5.

[31] — , "Max-min fairness linear transceiver design for a multi-user MIMO interference channel," IEEE Trans. Signal Process., vol. 61, no. 9, pp. 2413-2423, May 2013.

[32] M. Razaviyayn, M. Hong, and Z.-Q. Luo, "Linear transceiver design for a MIMO interfering broadcast channel achieving max-min fairness," Signal Process., vol. 93, no. 12, pp. 3327-3340, Dec. 2013.

[33] A. Wiesel, Y. C. Eldar, and S. Shamai, "Linear precoding via conic optimization for fixed MIMO receivers," IEEE Trans. Signal Process., vol. 54, no. 1, pp. 161-176, Jan. 2006.

[34] Y.-F. Liu, M. Hong, and Y.-H. Dai, "Max-min fairness linear transceiver design problem for a multi-user SIMO interference channel is polynomial time solvable," IEEE Signal Process. Lett., vol. 20, no. 1, pp. 27-30, Jan. 2013. 